\title{
Ansatz eines holistischen Datenstamms zur modellbasierten, domänenübergreifenden Nutzung in der Produktentstehung
}

\author{
Markus C. Berschik ${ }^{1 *}$, Fabian Laukotka ${ }^{1}$, Dieter Krause ${ }^{1}$ \\ ${ }^{1}$ Institute of Product Development and Mechanical Engineering Design (PKT), Hamburg University of Technology \\ (TUHH), Germany \\ * Korrespondierender Autor: \\ Markus Berschik \\ Technische Universität Hamburg \\ Institut für Produktentwicklung und Konstruktionstechnik \\ Denickestraße 17, 21271 Hamburg \\ Telefon: 040428782150 \\ Mail: markus.berschik@tuhh.de
}

\begin{abstract}
The current rise of interdisciplinarity in product creation leads to data sets that are characterised by different point of views which often come with inconsistencies. Furthermore, there is a trend towards using such data sets for innovative data-based product development processes, which require a holistic data representation. However, inconsistencies exacerbate these efforts. This paper presents an approach for a holistic data base for product creation that enables different point of views while also allowing for traceability and uniqueness. This approach uses model-based systems engineering techniques and consists of a model with two levels of detail, which allow for different point of views to coexist on a specific level while being connected on a generic level.
\end{abstract}

\section{Keywords}

product creation, point of view, inconsistencies, holistic data base, MBSE 


\section{Einleitung}

Bei der Entwicklung von mechatronischen Produkten fallen über die Produktlebensphasen hinweg Daten mit hohen interdisziplinären Abhängigkeiten an. Innerhalb der Produktlebensphasen gibt es verschiede Domänen in denen gearbeitete wird. Sowohl innerhalb als auch über die Domänen hinweg werden dabei unterschiedlichste Methoden angewendet. Die verschiedenen Domänen spiegeln sich auch in den Produktportfolios der verwendeten softwarebasierten Autorensysteme, wie beispielsweise CAD, FEM oder CAM Programmen, wider. Innerhalb dieser Domänen werden die spezifischen Daten durch die Programme gut gehandhabt, aber über die Anwendungsbereiche und Programme hinweg ist eine Nachverfolgbarkeit insbesondere von Metadaten jedoch sehr schwierig.

Ähnlich verhält es sich bei den verschiedenen Methoden, die meist einen spezifischen Blick auf ein Produkt haben. Bei der Entwicklung neuer Methoden erfolgt diese meist aus einem spezifischen Bedarf und einem definierten Blick auf das Problem [1]. Viele dieser Methoden stammen dabei aus einer Zeit in der die Interdisziplinarität von Produkten noch deutlich weniger ausgeprägt war, als sie es heute sind [2]. Daraus ergibt sich, dass bei der heutigen Anwendung von verschiedenen Methoden, die Kompatibilität zwischen diesen nicht unbedingt gegeben ist.

Es ist zu beobachten, dass sich die Forschung und Entwicklung von Methoden aber auch Autorensysteme von einer einst dokumentenbasierend, über eine prozessbasierend hin zu einer datenbasierten Entwicklung verlagert. [3, 4]. Für eine solche datenbasierende Entwicklung hat die Forschung gezeigt, dass das Datenmanagement mit Hilfe des MBSE für die einzelnen Produktlebensphasen mit ihren Herausforderungen eine sehr gute Unterstützung bieten kann [3].

Aktuelle Forschungsprojekte mit Partnern in der Luftfahrt greifen dies auf, indem der MBSE Ansatz genutzt werden soll, um die verwendeten Daten in der Produktentstehung besser zu verknüpfen. Dabei zeigt sich, dass modell-basierte Ansätze innerhalb von einzelnen Domänen zunehmend eingesetzt werden. Der holistische Einsatz wird allerdings dadurch erschwert, dass die verschiedenen Domänen und Methoden zu unterschiedlichen Blickwinkeln auf das Produkt haben, die miteinander nicht vollkommen kompatibel sind.

\section{Forschungsproblem und Forschungsziel}

Werden Modelle für die Beschreibung von Daten und Datenzusammenhängen verwendet, so sind sie immer ein Abbild eines Originals (Abbildungsmerkmal), abstrahieren das Abbild von der Realität (Verkürzungsmerkmal) und sind für einen bestimmten Zweck erstellt worden (pragmatisches Merkmal) [5]. Diese Hauptmerkmale bilden die Grundlage für die Betrachtung von verschiedenen Modellen. Besonders wichtig ist dabei die Ausprägung des Verkürzungsmerkmals, die im Folgenden als Level of Detail bezeichnen wird. Weiterhin wird das pragmatische Merkmal, das die Ausrichtung des Modells vorgibt, im Folgenden als Sichtweise bezeichnet. Innerhalb der Produktentstehung existieren, beispielsweise durch unterschiedliche Domänen oder verwendete Methoden, verschiedene Treiber von Sichtweisen auf ein Produktmodell. Auch unterschiedliche Methoden in der gleichen Domäne können dabei unterschiedliche Sichtweisen bewirken. Verschiedene Sichtweisen auf dasselbe Produkt, führen zu einer erschwerten Umsetzung der Grundidee von modell-basierten Ansätzen - der Nachverfolgbarkeit - da die Single Source of Truth (SSoT) schwieriger zu handhaben ist. Dieses Problem ist darauf zurückzuführen, dass unterschiedliche Sichtweisen oftmals dasselbe meinen, aber unterschiedlich darüber sprechen oder individuelle Parameter benutzen, die übergreifend betrachtet jedoch zusammenhängen. Als Resultat werden gleiche Daten an unterschiedlichen Stellen abgelegt oder unterschiedlich bezeichnet. Es entsteht eine Multiple Source of Truth und die Eindeutigkeit sowie Nachverfolgbarkeit ist nicht mehr möglich. Gleichzeitig liegt das Bestreben jedoch darin, nicht eine beliebige Vielzahl an individuellen 
Ansammlungen von Daten über das gleiche Produkt zu haben, sondern vielmehr alle relevanten Daten in einem gemeinsamen Datensatz zusammenzuführen.

Beispielhaft können in der Produktentwicklung die Domänen des Maschinebaus und der Elektrotechnik betrachtet werden. In beiden Domänen wird das Produkt unterschiedlich beschrieben. Selbst wenn der gleiche Parameter verwendet wird, können diese in anderen Zusammenhängen verwendet werden. Dies zeigt sich vereinfacht am Beispiel eines Elektromotors in Bild 1. Auch wenn beide Domänen den gleichen Elektromotor beschreiben, sind die Umsetzung, Betrachtungen und Dokumentationen in beiden Sichtweisen auf den ersten Blick unterschiedlich, wie auch durch die unterschiedlichen HintergrundVisualisierungen der Elektronik (links) und Mechanik (rechts) angedeutet wird. Dennoch fallen im Produktentstehungsprozess Daten an, die über die Sichtweisen hinweg verknüpft werden können. Eine nähere Ausführung dieses Beispiels ist zudem in Kapitel 5.1 zu finden.

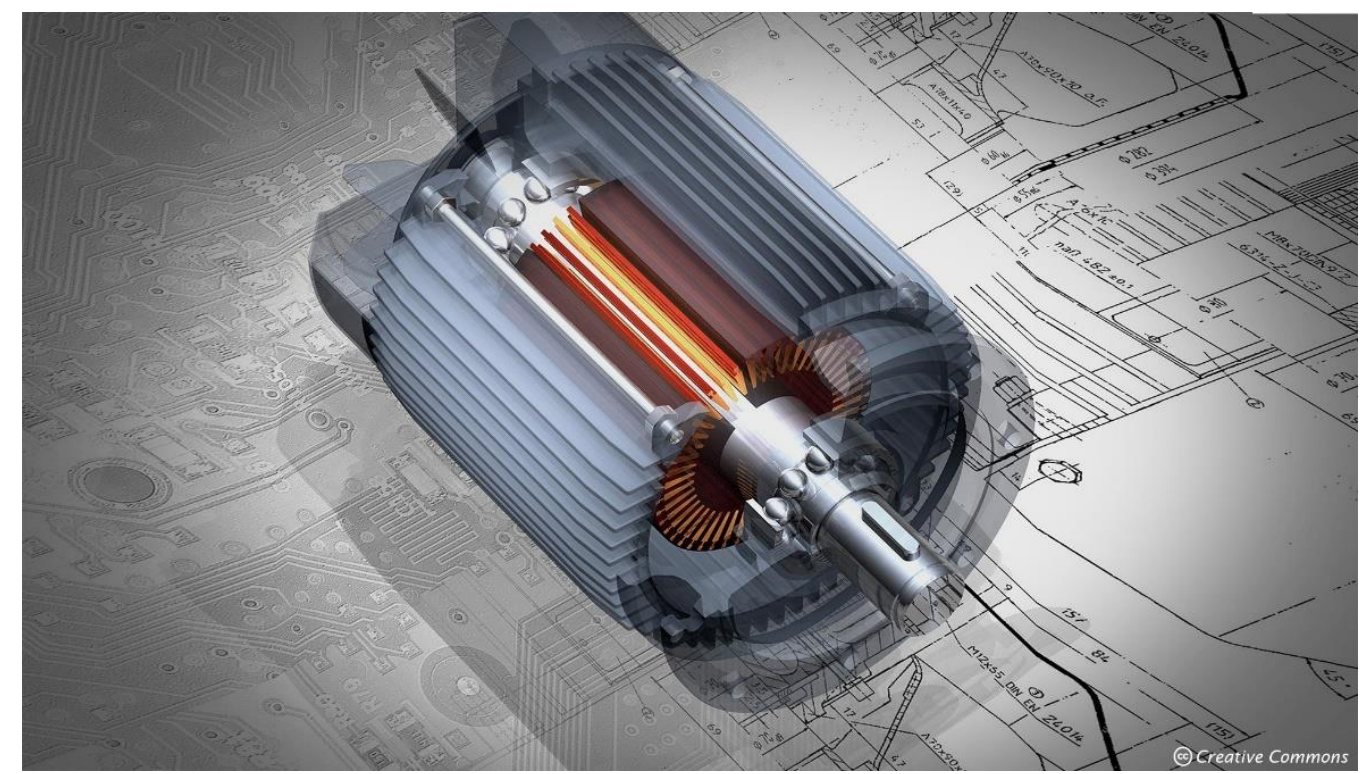

Bild 1: Beispiel eines Elektromotors als mechatronisches Produkt, mit den Anwendungsbereichen der verschiedenen Domänen, Elektronik und Mechanik, dargestellt durch Hintergrund-Visualisierungen

Eine ähnliche Herausforderung ist auch bei der Betrachtung von Methoden zu erkennen. In der Produktentstehung werden oftmals unterschiedliche Methoden zeitlich versetzt oder parallel für unterschiedliche Bereiche der Produktentstehung verwendet.

Betrachtet man z.B. den Integrierten PKT-Ansatz zur Entwicklung modularer Produktfamilien (Integrierter PKT-Ansatz), welcher einen adaptiv zusammenstellbaren Methodenbaukasten bereitstellt, um die unternehmensinterne Vielfalt der Komponenten und Prozesse zu minimieren und gleichzeitig die für den Kunden sichtbare Nachfragevarianz uneingeschränkt zu bedienen. Die bereitgestellten Methoden betrachten das Produkt dabei sowohl produktstrategisch als auch funktional. [2]

Ein anderes Beispiel einer Methode in der Produktentwicklung ist die VDI 2221 zur Entwicklung technischer Produkte und Systeme, welche eine grundlegende Richtlinie zur interdisziplinären Entwicklung von Produkten darstellt [6]. In beiden Entwicklungsansätzen wird auf unterschiedlichen Ebenen über gleiche Daten gesprochen. Gleichzeitig werden dabei unterschiedliche Effekte betrachtet, die sich über die Sichtweisen hinweg teilweise beeinflussen. So wird im Integrierten PKT-Ansatz von Modulen und Komponenten gesprochen, während die VDI 2221 Produkte in Bauteile und Baugruppen unterteilt. [2, 6]

Für verschiedene Anwendungsfälle sind die jeweiligen Sichtweisen der Methoden passend, die in den Methoden behandelten Daten sind damit aber nicht immer vollständig zueinander kompatibel. Auch wenn diese Methoden nicht zwangsläufig parallel oder direkt aufeinander 
aufbauend verwendet werden, kann es im Laufe des Produktlebenszyklus dazu kommen, dass unterschiedliche Methoden den gleichen Datensatz verwenden. Dies ist beispielsweise bei der zunehmenden Konzipierung von Ansätzen mit sogenannten Digitalen Zwillingen vielfach zu beobachten [7]. Weiterführend können damit in Zukunft auch Machine-Learning Ansätze in der Produktentstehung ermöglicht werden, da für diese genau solche holistischen digital verarbeitbare Datensätze benötig werden.

Eine weitere Herausforderung stellt die bisherige Anwendung von MBSE in unterschiedlichen Domänen oder Methoden dar. Bisher entstehen einzelne Modelle mit Fokus auf eine explizite Sichtweise. Eine übergeordnete Einordnung wird dabei zumeist vernachlässigt. Dies führt insgesamt dazu, dass Modelle durch ihre fehlende Kompatibilität zu anderen Modellen nicht oder nur kaum für ähnliche Anwendungen wiederverwendet werden können [3]. Dabei zeigt sich, dass eine fokussierte übergreifende Modellierung von komplizierten Produkten zu einer besseren und kostengünstigeren Entwicklung beiträgt [8].

Aus den beschriebenen Herausforderungen resultiert folgende Forschungsfrage:

- Wie kann innerhalb des modell-basierten Arbeitens eine interdisziplinäre Grundlage geschaffen werden, welches die datengetriebene Durchgängigkeit über verschiedene Sichtweisen hinweg ermöglicht?

Neben der damit erreichbaren Kompatibilität der Daten an sich, ist auch die Integration der Modelle in bestehende Workflows von Bedeutung, um den holistischen Einsatz weitere zu Unterstützen. Ziel dieses Papers soll es daher sein, einen Ansatz für eine gemeinsame Grundlage in Form eines Datenstamms, für die datengetriebene Abbildung eines Produktes vorzustellen. Mit diesem soll die Herausforderung der Kompatibilität von verschiedenen Sichtweisen bei der Nutzung von modell-basierten Ansätzen verbessert werden.

Ein Datenstamm beschreibt in diesem Paper die Ansammlung von Datenelementen aus den verschiedenen Sichtweisen und stellt damit eine Art Baukasten für die Verwendung im Zuge der Modellierung durch den Benutzer dar. Ein Datenelement kann dabei, z.B. eine Drehzahl, Spannung aber auch Stückzahl oder Kosten abbilden. Diese Datenelemente werden mit entsprechenden Informationen wie 2000 Umdrehungen pro Minute, 120 Volt aber auch 15 Stück oder $200 €$ gefüllt.

Da Schnittstellen für die holistische Verwendung des Datenstamms von elementarer Bedeutung sind, wird dieser Aspekt kurz im folgenden Kapitel beschrieben. Nach dem Beschreiben der Vorgehensweise in Kapitel 4 wird sich in Kapitel 5 auf den Datenstamm, als Antwort auf die Forschungsfrage fokussiert.

\section{Der Weg von MBSE aus dem Frontend ins Backend}

Für das Datenmanagement von Produkten hat sich die Nutzung von MBSE-Ansätzen als gute Möglichkeit herausgestellt. So ist in den letzten Jahren eine Zunahme der Verwendung von MBSE und damit auch von Systemmodellen zu beobachten. Aktuell erfolgt die Modellierung und damit auch insbesondere der Zugriff auf die in den Modellen hinterlegten Daten durch die Verwendung von Modellierungssoftware. Auch wenn diese in der Regel die Möglichkeit bieten Modelldaten beispielsweise als CSV-Dateien zu exportieren und geänderte Werte aus diesen Dateien zu importieren [9], ist dazu ein aktives Benutzen der entsprechenden Programme notwendig. Dies führt dazu, dass in den Arbeitsschritten des Anwenders neben dem eigentlich verwendeten Programm, beispielsweise eine CADAnwendung, parallel auch die Modellierungssoftware verwendet werden muss. Dies erfordert neben der Software an sich auch die dafür notwendigen MBSE- bzw. SysML Kenntnisse. Besser wäre es, wenn dedizierte Systemingenieure das MBSE-Grundgerüst erstellen und verwalten, der altägliche Datenaustausch mit den Modellen allerdings ohne detaillierte 
Kenntnisse erfolgten kann. Dafür ist eine Interaktion mit diesen Modellen notwendig, die idealerweise weitgehend ohne direkte Benutzerinteraktion erfolgt. Die dafür notwendigen Schnittstellen (Application Programming Interfaces: APIs) für die automatisierte, definierte Interaktion zwischen zwei Programmen gibt es bereits seit längerer Zeit und sind bereits Bestandteil vieler allertäglichen Prozesse.

In den aktuell laufenden Arbeiten an SysML V2 wird unter anderem genau dieser Ansatz verfolgt, indem ein offener Standard für Schnittstellenzugriffe auf Systemmodelle via APIs definiert werden soll [10]. Erste Prototypen dieses Standards zeigen dies bereits, in dem sie die Daten von Systemmodellen über eine entsprechende Plattform und APIs für andere Programme zur Verfügung stellen. Durch den offenen Standard wird zudem ermöglicht, individuelle Lösungen oder Plug-Ins beispielsweise für bestehende CAD-Softwares zu entwickeln, die ebenfalls diese Plattform verwenden.

Der geplante Schnittstellen-Standard von SysML V2 stellt damit einen Enabler für den Weg von MBSE als Frontend-Anwendung hin zu einer Datenverwaltung durch MBSE im Backend dar. Der Weg bis zur tatsächlichen Umsetzung ist jedoch mit dem Schnittstellenstandard nicht vollendet, da dieser noch durch die entsprechenden Programme (oder Addins für diese) implementiert werden muss. Durch den offenen Standard sind allerdings individuelle Adaptionen oder Lösungen möglich. Auf den ersten Blick scheint dieser Ansatz in Konkurrenz zu bereits etablierten PDM-/PLM-Systemen zu stehen. Tatsächlich ergänzen sich MBSE und PLM jedoch, da beide Systeme unterschiedliche Fokusse und Stärken haben und auch vermehrt in Kombination verwendet werden [11, 12].

\section{Vorgehensweise}

Im Zuge eines aktuellen Forschungsprojekts ist der Bedarf ersichtlich geworden, Daten über die verschiedenen Sichtweisen der Produktentstehung hinweg einheitlich zu dokumentieren und konsistent zu verknüpfen. Dafür werden in einem ersten Schritt die unterschiedlichen Domänen und Methoden als Sichtweisen dahingehend untersucht, welche Daten übergreifend von Relevanz sind und daher in dem gemeinsamen Datenstamm definiert sein müssen. In einem zweiten Schritt werden mögliche Zusammenhänge oder Abhängigkeiten zwischen den identifizierten Daten analysiert und modellbasiert dokumentiert. Zur Erprobung des Ansatzes wird dieser anschließend an vereinfachten Beispielen durchgeführt und die Modellierung dabei mit der Software Cameo Systems Modeler umgesetzt. Mit Hilfe dieser Software werden schließlich Zusammenhänge zwischen den Modellen der verschiedenen Sichtweisen automatisch analysiert und Effektketten aufgezeigt.

\section{Ergebnisse und Diskussion}

In diesem Kapitel wird der Ansatz eines holistischen Datenstamms zur modellbasierten, domänenübergreifenden Nutzung in der Produktentstehung näher beschrieben. Dazu wird zunächst die Grundidee des Ansatzes vorgestellt, bevor er am Beispiel der Domäne als Sichtweisentreiber in Kapitel 5.1 näher beschrieben wird. Anschließend erfolgt in Kapitel 5.2 eine Darstellung einer möglichen Umsetzung in dem Programm Cameo Systems Modeler am Beispiel der Methoden als Sichtweisentreiber.

Die Entwicklung eines Produktes setzt sich aus der Betrachtung einzelner Sichtweisen zusammen, die sich auch in verschiedenen Modellen äußern. Diese führen jedoch dazu, dass keine einheitliche und vor allem übergreifende Modellierung möglich ist. Vorteile, wie die bessere Darstellung von Verknüpfungen von Daten, deren Nachverfolgbarkeit insbesondere bei Änderungen und die damit verbundene Konsistenz der Daten, die durch eine Verwendung von modell-basierten Ansätzen entstehen, können nicht vollkommen ausgenutzt werden.

Durch den momentanen Trend zur datenbasierten Entwicklung entsteht jedoch eine Lösung, für die fundamentalen Herausforderungen der verschiedenen Sichtweisen. Ein 
übergeordnetes Datenmanagement bezieht sich dabei, auf die verwendeten Daten in den Domänen, den unterschiedlichen Methoden und den Autorensystemen, die zur Entwicklung genutzt werden. Es wird nicht versucht die einzelnen Sichtweisen im Prozess zu vereinheitlichen, sondern auf einer darüber liegenden Ebenen und damit den gesamtheitlich verwendeten Daten anzusetzen. Mithilfe eines übergeordneten Modells sollen die Daten nachhaltig und konsistent abgelegt werden. Durch eine ergänzende übergreifende Modellierung können Wechselwirkungen und Abhängigkeit zwischen den Sichtweisen frühzeitig erkannt und abgebildet werden.

Selbst wenn bei kleineren Beispielen eine Modellierung in SysML noch nicht von Nöten ist, hilft eine saubere, übergeordnete Modellstruktur bei einer späteren Skalierung zu komplexeren Produkten. Aus diesem Grund wird im Folgenden der zuvor bereits beschriebene Elektromotor erneut als einfaches Beispiel gewählt, um den Aufbau und die Umsetzung des Datenstamms zu erklären.

\subsection{Domänen als Sichtweisentreiber}

Wie zuvor beschrieben führen unter anderem die verschiedenen Domänen in der Produktentstehung zu unterschiedlichen Sichtweisen auf ein Produkt. So werden beispielsweise spezifische Autorensysteme, für technische Zeichnungen, elektrische Schaltpläne oder den technischen Einkauf, genutzt. Zusätzlich liegt dabei der Fokus ggf. auf unterschiedlichen Bereichen oder Aspekten des Produkts.

Bei Arbeiten in jeder Sichtweise entstehen Modelle, die die relevanten Daten aus ihrem Blickwinkel beschreiben. Innerhalb einer Sichtweise sind die Daten dabei in der Regel kompatibel und eine Nachverfolgbarkeit lassen sich mit heutigen Mitteln einfach umsetzen.

Da es jedoch schwierig sein wird, sich in allen betroffenen Domänen und deren Mitarbeiter sowie Vorgehensweisen auf einen gemeinsamen Standard der Modellierung und ein einheitliches Wording zu einigen, ist der hier präsentierte Ansatz, stattdessen einen gemeinsamen Nenner zu finden und über diesen die verschiedenen Daten der Sichtweisen kompatibel zueinander zu machen. Neben der Spezifischen Datenebene, in denen die verschiedenen Sichtweisen wie gewohnt arbeiten können, sieht dieser Ansatz daher eine zusätzliche Generische Datenebene vor, in der unter anderem die Zusammenhänge zwischen Daten verschiedener Sichtweisen beschrieben sind.

Diese Situation ist vereinfacht in Bild 2 illustriert. In der linken Darstellung spannen die verschiedenen Sichtweisen Gebiete auf, in denen die relevanten Datenelemente der Sichtweise verwendet werden können. Die einzelnen Bereiche passen dabei nicht nahtlos zueinander und überlagern sich an einigen Stellen, während es an anderen Stellen auch zu Lücken zwischen ihnen kommt. Insgesamt ist eine nahtlose Komptabilität zwischen Sichtweisen und damit den darin enthaltenen Datenelementen nicht gegeben.
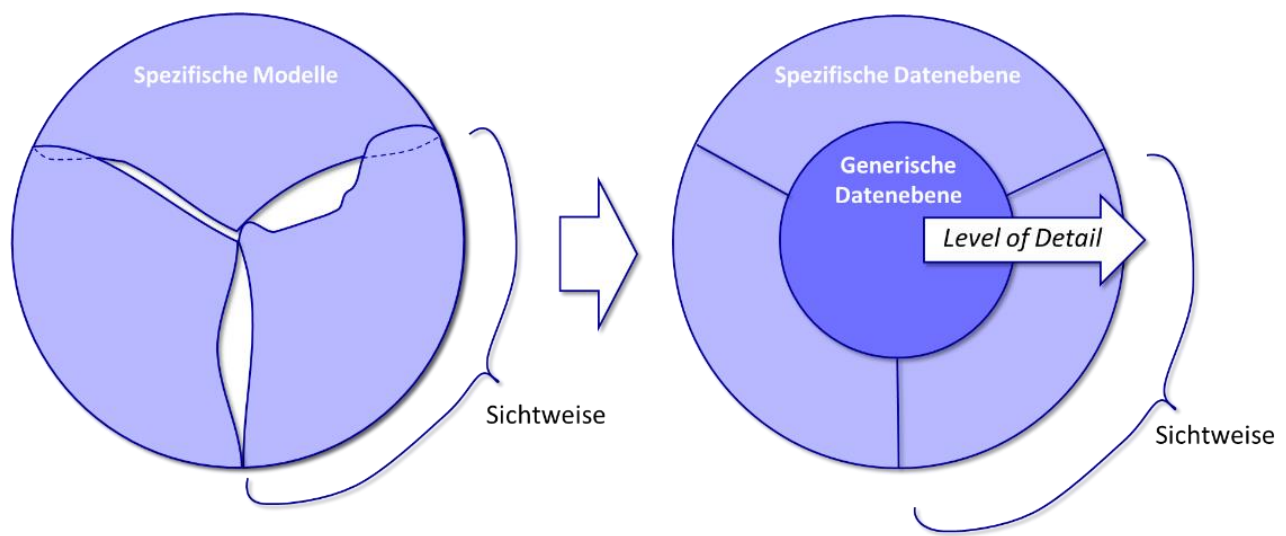

Bild 2: Abstrakte Visualisierung des holistischen Datenstamms bestehend aus Spezifischer und Generischer Datenebene zur Ermöglichung einer Kompatibilität verschiedener Sichtweisen 
In der rechten Darstellung ist zusätzlich zentral die Generische Datenebene aus dem vorgestellten Ansatz dargestellt. Sie stellt wie zuvor beschrieben den gemeinsamen Nenner der Sichtweisen dar und führt dazu, dass die Datenelemente aus den Sichtweisen über dieses zentrale Element in Verbindung gebracht werden können.

Um das Beispiel des Elektromotors aufzugreifen, lässt sich beispielsweise innerhalb eines Spezifischen Modells in der Domäne der Elektrotechnik, der Schaltplan mit den verwendeten Bauteilen einordnen. Ähnlich dazu können die Metadaten von CAD-Modellen oder die Struktur und das Verhalten einzelner Bauteile in einem Spezifischen Modell der Domäne des Maschinenbaus eingeordnet werden.

Durch die Beschreibungen auf der Generischen Ebene lassen sich beispielsweise die Auswirkung der Änderung der Wicklungsanzahl in der Elektrotechnik über daraus resultierende Drehzahlen oder abhängige Mindestdurchmesser auf Parameter des Maschinenbaus wie Lagerbelastungen direkt nachverfolgen. Der Datenstamm beinhaltet in diesem Fall also auch technische Datenelemente, die unter anderem relevante physikalische Zusammenhänge beschreiben. Erst dadurch wird ermöglicht, dass daraus resultierende Abhängigkeiten identifiziert und für die Nachverfolgbarkeit von Änderungen und entsprechenden Effektketten verwendet werden können. Gerade bei komplexeren Produkten mit mehreren Abhängigkeiten, die auch aufeinander aufbauen, lässt sich auf diese Art und Weise eine sichtweisenübergreifende Modellierung umsetzen.

\subsection{Methoden als Sichtweisentreiber}

Ähnlich wie die verschiedenen Domänen treiben auch verschiedene Methoden neue Sichtweisen auf das Modell eines Produkts. Jede Methode ist aus einem bestimmten Anlass und zu einem bestimmten Zweck erstellt worden [1]. Dementsprechend geben die Methoden einen spezifischen Blick auf das Produkt und damit auch auf die Daten wieder. Die verwendeten Visualisierungen und Werkzeuge die dem Entwickler an die Hand gegeben werden, fördern damit auch hier die klare Trennung der Sichtweise.

Im Folgenden werden zwei beispielhafte Methoden und ihre Modelle vorgestellt, um den Nutzen des holistischen Datenstamms weiter zu erklären. Mithilfe einer Methode wird dem Entwickler eine Vorgehensweise zur Verfügung gestellt mit der er Daten, die für die Entwicklung benötigt werden, in eine geeignete Beziehung setzen kann. Die modellbasierte Umsetzung von angewendeten Methoden, die nicht für die Nutzung in einer Modellierungssprache entwickelt wurden, lassen sich zumeist anhand ihrer beschriebenen einzelnen Methodenschritte abbilden. Dabei bildet jeder Methodenschritt ein eigenes Modell auf der Spezifischen Datenebene des holistischen Datenstamms. Hauptaugenmerk bei der Überführung liegt dabei auf der Abbildung der Daten und ihrer Verknüpfungen. Insgesamt können so nebeneinander verschiedene Sichtweisen abgebildet werden. Um die verschiedenen Sichtweisen zu einem holistischen Datenstamm zu verbinden, wird auch hier der Generischen Datenebene verwendet.

Anhand des PKT-Ansatzes und der VDI 2221, als zwei anerkannte Entwicklungsmethoden, die jedoch unterschiedlichen Fokussierungen in der Produktentwicklung aufweisen, wird der beschriebene Aufbau des Datenstamms betrachtet. Dazu wird die Modellierungssprache SysML im Cameo Systems Modeler verwendet. Die einzelnen Datenelemente werden durch verschiedene Stereotypen auf der Generischen Datenebene modelliert. Jeder Methodenschritt zeigt einzelne Verknüpfungen von Datenelementen auf, die insgesamt die Datenlage eines Produktes detaillierter beschreiben. Nachdem diese Modelle mit ihren Datenelementen auf der Spezifischen Datenebene modelliert sind, können in einem folgenden Schritt die übergeordneten Zusammenhänge auf der Generischen Datenebene modelliert werden. So lassen sich, ähnlich wie mit den physikalischen Zusammenhängen bei den Domänen, alle auf der Spezifischen Datenebene verwendete Datenelemente in einem zusammenfassenden Modell in Relation bringen. Dies erfolgt auf Basis der angewendeten Stereotypen. 
Generell lässt sich festhalten, dass immer dann, wenn verschiedene Sichtweisen aufeinandertreffen, die verwendeten Datenelemente der gemeinsame Nenner für ein holistisches und sichtweisenübergreifendes Modell sind. Die verwendeten Datenelemente können dabei teilweise Verknüpfungen haben, an anderen Stellen jedoch stark voneinander unterschieden werden. Dennoch ist auch eine explizit nichtexistierende Verknüpfung eine wichtige Information für die übergeordnete Modellierung.

Eine exemplarische Umsetzung des Ansatzes in Form einer Modellierung mit SysML ist in Bild 3 dargestellt. Als Beispiel dient ein Staubsauger-Roboter mit elektrischem Bürstenantrieb, wobei der dazu verwendete Elektromotor zwar die vorherigen Beispiele aufgreift, in diesem Anwendungsfall jedoch nicht derselbe Elektromotor wie zuvor ist. In der Modellierungsoberfläche sind die beschriebenen Aspekte des Ansatzes zu sehen. Links sind die Datenelemente und Modelle der Spezifischen (1) sowie die der Generischen Datenebene (2) zu finden. Die Modellansicht unterteilt sich in zwei Ansichten, in denen jeweils ein Modell einer Methode mit unterschiedlichen Sichtweisen der Struktur des Produkts dargestellt wird. Auf der linken Seite beschreibt der Integrierte PKT-Ansatz die Struktur über Komponenten und Module (3), auf der rechten Seite beschreibt die Sichtweise der VDI 2221 Bauteile und Baugruppen (4). Obwohl beide Sichtweise Daten und Informationen des StaubsaugerRoboters abbilden, haben sie dabei einen unterschiedlichen Fokus. Die Visualisierung soll hier nur den generellen Aufbau und weniger die Daten der Modellierung verdeutlichen.

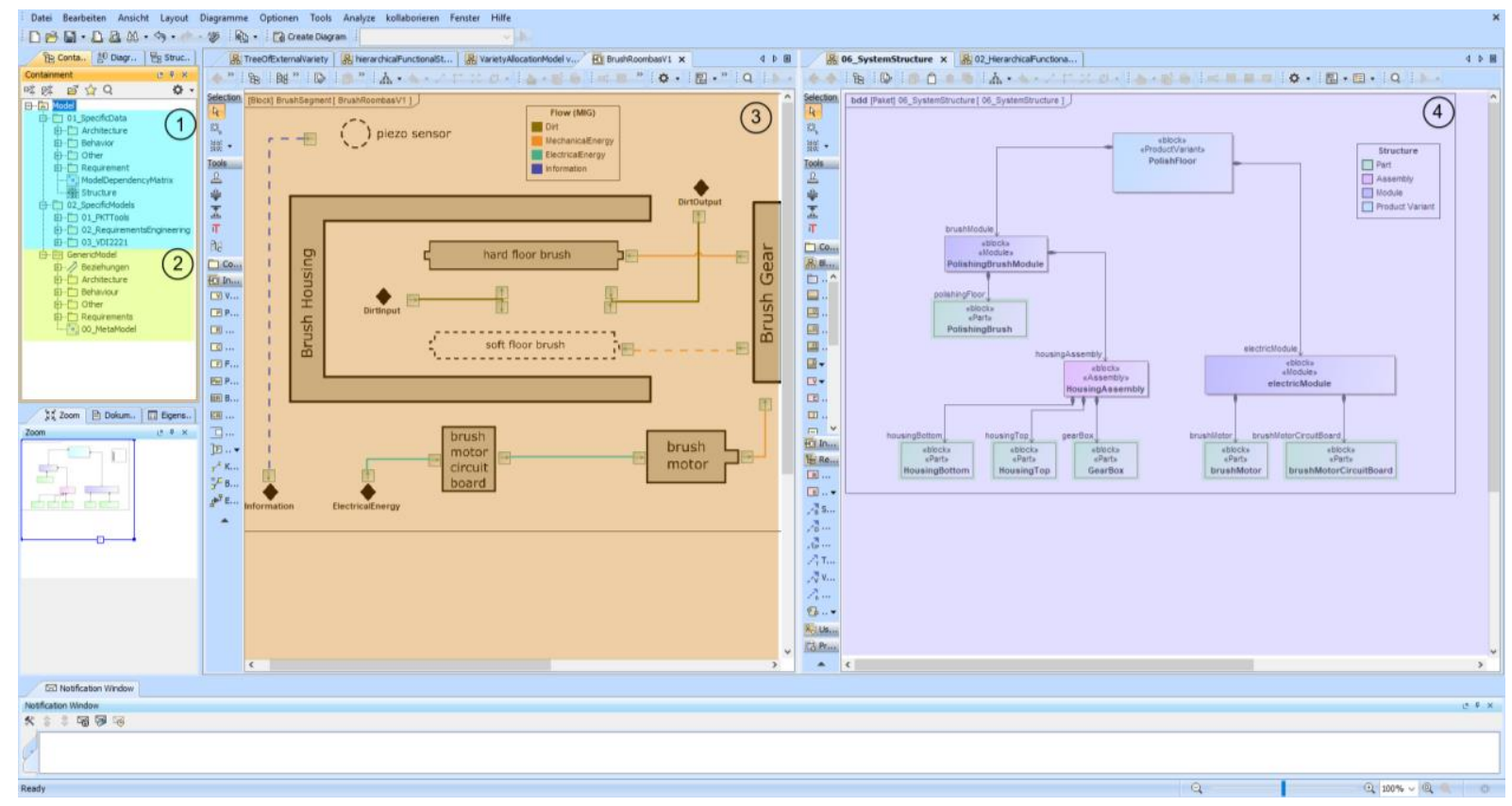

Bild 3: Exemplarische Umsetzung des Ansatzes in Form einer SysML-Modellierung der Produktstruktur eines Staubsauger-Roboters aus unterschiedlichen Sichtweisen

Auch wenn die beiden Methoden nicht zeitgleich auf dasselbe Produkt angewendet werden, kann es im Laufe der Produktentstehung oder des gesamten Produktlebenszyklus auch über mehrere Produktgenerationen hinweg dazu kommen, dass beide Methoden auf dieselben Daten zugreifen. Für eine übergeordnete Produktbeschreibung lassen sich diese Fokussierungen auf bestimmte Datenelemente jedoch nicht auflösen, da diese innerhalb einer Sichtweise sinnvoll, wenn nicht sogar notwendig, ist. Die Darstellung der Produktstruktur im Integrierten PKT-Ansatz folgt beispielsweise einem definierten Wording mit sehr Spezifischen Datenelementen und auch einem definierten visuellen Schema. Die Lösung stellt daher der beschriebene Schritt über eine übergeordnete Ebene dar, die die Zusammenhänge und 
Verknüpfungen definiert und dadurch eine Art „Verkehrssprache“ etabliert. Diese Verknüpfungen können so zum Beispiel zeigen, das Bauteil und Baugruppen in Komponenten aufgelöst werden. Mit dieser Information auf der Generischen Datenebene können so im späteren Verlauf, wenn sich beispielsweise Änderungen an einem Bauteil vorgenommen werden, anhand des holistischen Datenstamm auf die dabei mitveränderte Baugruppe oder Komponente geschlossen werden.

Durch geeignete Modellierungssoftwares lassen sich bei der Verwendung des Datenstamms automatisch solche Effektketten aufzeigen und darstellen. Bild 4 zeigt so eine automatisch erzeugte Darstellung, in der die Zusammenhänge über verschiedene Sichtweisen hinweg analysiert und nachvollzogen werden kann. Ausgehend von einer Änderung einer Anforderung lässt sich gemäß den modellierten Datenzusammenhängen des Integrierten PKT-Ansatzes auf das betroffene Wirkprinzip schließen. Parallel dazu lassen sich in der VDI 2221 Abhängigkeiten von Wirkprinzipien auf Bauteile ziehen. Die Wirkprinzipien der einzelnen Sichtweisen sind dabei zunächst unabhängig voneinander. Ohne eine Kompatibilität wäre an dieser Stelle keine weitere Nachverfolgbarkeit möglich. Durch die definierten Zusammenhänge auf Generischer Datenebene lassen sich die Auswirkungen der Anforderungsänderung jedoch aus der Sichtweise des Integrierten PKT-Ansatzes in die Sichtweise der VDI2221 weiterfolgen und somit das betroffene Bauteil identifizieren.

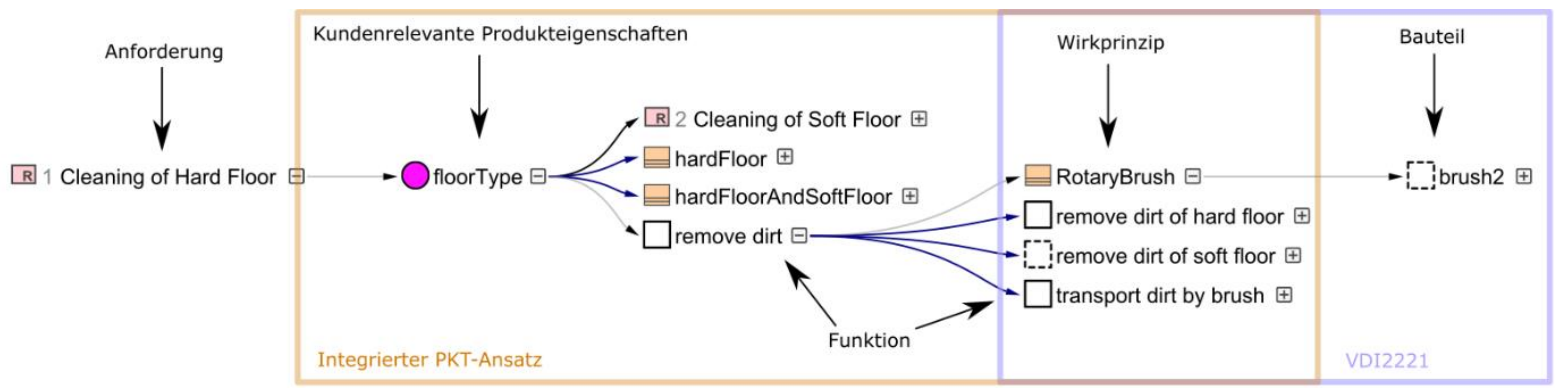

Bild 4: Automatisch erzeugte Darstellung ausgewählter Zusammenhänger im holistischen Datenstamm über verschiedene Sichtweisen hinweg

An dieser Stelle könnte dem Entwickler, aufgrund der Nutzung des holistischen Datenstamms, eine Meldung über existierende Änderungen gegeben werden und so das über Sichtweisen hinweg angestoßene Änderungsmanagement maßgeblich verbessert werden. Dies mag bei der Komplexität des gewählten Beispiels an dieser Stelle auch noch von Hand möglich sein, bei Produkten mit komplizierterer Produktstruktur oder anderen gekoppelten Sichtweisen wird eine solche durchgehende Nachverfolgbarkeit jedoch zunehmend schwerer ohne entsprechende Unterstützung realisierbar.

\section{Zusammenfassung und Ausblick}

Zusammenfassend lässt sich feststellen, dass für heutige existierenden Produkte, die eine Vielzahl an verschiedenen Arbeitsbereichen oder Domänen also Sichtweisen benötigen, es nicht möglich sein wird eine einheitliche Lösung in Form eines Modells zu finden auf das sich alle Beteiligten einigen und das allen Belangen gerecht wird. Daher ist das Ziel eine Modellebene zu schaffen, die die etablierten Modelle miteinander kompatibel macht. Diese Modellebene wurde als der Kern des Datenstamms eingeführt und nutzt die verwendeten Datenelemente als gemeinsamer Nenner für die übergeordnete Modellierung. Auf Basis der verwendeten Datenelemente können Zusammenhängen aber auch Differenzen, die ansonsten nicht deutlich geworden wäre, modelliert werden.

Ändert sich ein Datenelement in der einen Sichtweise so kann über den Datenstamm nachverfolgt werden, wo Verknüpfungen zu anderen Datenelemente in anderen Sichtweisen 
existieren und diese dann gegebenenfalls informiert bzw. geändert werden. Somit wird eine Nachverfolgbarkeit über die Sichtweisen hinweg ermöglicht.

Der beschriebene Datenstamm kann insgesamt als eine Art Baukasten verstanden werden, in dem Datenelemente mit ihren Verknüpfungen abgelegt sind. Das Ziel dieses Baukastens ist die eindeutige Definition von Datenelementen. Jedes Datenelement soll demzufolge nur einmal im Datenstamm vorkommen, insbesondere auch dann, wenn das Datenelement in mehreren Sichtweisen verwendet wird. Um den Datenstamm so umzusetzen, bedarf es einem Systemingenieur, der im Backend die einzelnen Sichtweisen aufeinander abstimmt. Dazu müssen bei der Einführung neuer Sichtweisen die verwendeten Datenelemente analysiert und holistisch integriert werden. Parallel dazu müssen die Verknüpfungen zu den bereits auf der Generischen Datenebene existierenden Datenelementen gezogen werden. Nur so kann der Datenstamm als Basis für alle Sichtweisen eine Vorlage für die Datenelemente bieten.

In diesem Paper wurde der Ansatz anhand von vereinfachten Beispielen vorgestellt und erklärt. Für eine Überprüfung der Skalierbarkeit ist daher die Durchführung einer Case-Study, beispielsweise mit einem geeigneten Industriepartner ein denkbarer nächster Schritt. Ferner stellt dies möglicherweise eine Grundlage für die Entwicklung von entsprechender MachineLearning Ansätzen in der Produktentstehung dar.

Wenngleich der beschriebene Ansatz dafür sorgt, dass Daten auch über die verschiedenen Sichtweisen hinweg als Single Source of Truth verwendet werden können, bedarf es weiterer Arbeiten, um den Einsatz von Systemmodellen auch im Backend zu ermöglichen. Erst dadurch wird es möglich, dass dieser Datenstamm holistisch verwendet werden kann, ohne dass die Ingenieure in ihrer Sichtweise eingeschränkt werden. Sie arbeiten wie gewohnt während beispielsweise die verwendeten Autorensysteme automatisch Metadaten in das Backend speisen oder von dort auslesen. Der hier präsentierte Ansatz ist jedoch komplementär zu dieser vollautomatisierten Nutzung zu verstehen, da er auch bei manueller Interaktion durch den Benutzer die Datendurchgängigkeit und Konsistenz über Sichtweisen hinweg fördert. Es ist jedoch festzustellen, dass mit einer automatisierten Verwendung im Backend die zu erzielenden Effekte noch verstärkt werden können.

\section{Literaturverzeichnis}

[1] Blessing, Lucienne T. M.; Chakrabarti, Amaresh: DRM, a Design Research Methodology, Springer, Dordrecht, Heidelberg, London, New York, 2009.

[2] Krause, Dieter; Gebhardt, Nicolas: Methodische Entwicklung modularer Produktfamilien. Springer Verlag, Berlin, Heidelberg, 2018.

[3] Seiler, Florian M. et al.: MBSE zur Unterstützung der Produktentwicklung von modularen Produktarchitekturen, in Krause, Dieter; Hartwich, Tobias S.; Rennpferdt, Christoph (Hrsg.): Produktentwicklung und Konstruktionstechnik, Springer Verlag, Berlin, Heidelberg, 2020, pp. 111-134. https://doi.org/10.1007/978-3-662-62393-0_5

[4] Autodesk Inc.: Autodesk und Model Based Systems Engineering (MBSE), Vortrag, 2021.

[5] Stachowiak, Herbert: Allgemeine Modelltheorie. Springer-Verlag, Wien, New York, 1973.

[6] VDI-Richtlinie 2221: Methodik zum Entwickeln und Konstruieren technischer Systeme und Produkte, VDI, Düsseldorf, 1993.

[7] Laukotka, Fabian; Hanna, Michael; Krause, Dieter: Digital Twins of Product Families in Aviation based on an MBSE-assisted approach, Procedia CIRP, Vol. 100, 2021, pp. 684-689. https://doi.org/10.1016/j.procir.2021.05.144

[8] Honour, Eric C.: Systems engineering return on investment, Dissertation, University of South Australia, 2013.

[9] Dasgupta, Sanjeev; NoMagic Inc.: Cameo Simulation Toolkit Documentation, online gestellt am 01.08.2016, https://docs.nomagic.com/display/CSTD184/CSV+Export, abgerufen am 20.06.2021.

[10] Object Management Group Inc.: Systems Modeling Language (SysML®) v2 Request For Proposal, 2017.

[11] Apperly, Hedley; PTC Inc.: Den Nutzen der vernetzten MBSE- und PLM Werkzeugkette erschließen, online gestellt am 13.12.2016, https://www.ptc.com/de/blogs/plm/tap-into-the-value-of-the-connected-mbse-andplm-tool-chain, abgerufen am 25.06.2021.

[12] Muggeo, Christian; Pfenning, Michael: Die Rolle von MBSE und PLM im Industrial Internet, In: Tag des Systems Engineering. 2015. 